\title{
Multifrequency-Coupled Oscillators for Distributed Multiagent Coordination
}

\author{
Manuel Castillo-Cagigal, Eduardo Matallanas, Félix Monasterio-Huelin, Estefanía Caamaño Martín, \\ and Álvaro Gutiérrez
}

\begin{abstract}
The coordination of multiagent systems in real environments receives considerable attention from research and industry. The design of coordination mechanisms should take into account the nature of the environment where the system is embedded. In this paper, the multiagent system is in an environment that features periodic properties. This environment is approached from a signal processing point of view to extract such properties. The coordination is performed by the proposed multifrequency-coupled oscillators (MuFCO) algorithm. It addresses the coordination of the multiagent system as a distributed collective synchronization mechanism. An operation example of MuFCO algorithm is shown, where it is used to coordinate consumptions in a smart grid. Thanks to the MuFCO algorithm, the multiagent system can be used to smooth the aggregated consumption of an electrical grid in a distributed way.
\end{abstract}

Index Terms-Collective synchronization, distributed coordination, multiagent system, smart grids.

\section{INTRODUCTION}

$\mathbf{T}$ HE COORDINATION of distributed multiagent systems is an active research field. The interaction between agents is a key factor in this coordination which can become highly complex depending on both internal and external factors. In practical applications, multiagent systems are usually embedded in real environments. In this case, the coordination of these systems may be strongly affected by different elements of the environment. Previous works have studied multiagent coordination with the presence of noise or disturbances [1]. For example, these effects have been studied in networked systems performing consensus algorithms [2], [3]. However, the environment may affect the coordination process, not only as noise or disturbance, but also to the nature of the coordination process itself. In such a case, the coordination between agents may include certain adaptation to variations of the environment where they are. This adaptive process may be designed so that the environment meets certain predefined characteristics or goals. This design procedure is typical in the design of artificial systems where there are several elements that interact with the environment [4]. To this end, information on the nature of the environment should be considered during the design process.

The dynamics of real environments is usually complex since it includes events of different nature. A specific case of use is the one in which the environment has periodic properties. This periodicity means that certain events appear in the environment with a specific frequency. Periodicity can originate from both natural and artificial phenomena, e.g., earth's rotation and revolution, tides, cyclic production processes, and customer demands. In this kind of environments, the multiagent system coordination may be addressed from the collective synchronization point of view. There is a wide variety of collective synchronization phenomena in which a system composed of a collective of elements suddenly locks to a common phase causing that the collective oscillates in unison. These phenomena have been observed and studied in biology and physics [5], [6]: networks of pacemaker cells in the heart [7], arrays of lasers [8], different self-organizing processes [9], etc. Synchronization has been previously used for multiagent coordination [10], [11]. For example, a new type of distributed consensus filters was designed based on the theory of synchronization and consensus in complex networks and systems in [2]. In [12], traveling groups of agents were produced using chorusing mechanisms. In [13], an algorithm inspired on fireflies synchronization was used to detect nonoperational robots in a swarm robotic system. Most works done on this field are focused on the dynamic behavior of a collective of oscillators without considering their integration in the environment. This paper differs from previous works in the inclusion of the periodic components of the environment directly in the synchronization process. Thereby, the coordination of the multiagent system with the environment can be approached as collective synchronization.

This paper proposes an algorithm which implements a multiagent system coordination in periodic environments. The environment is analyzed from the signal processing point of view to extract its periodic properties. The coordination is based on collective synchronization by including the effects 
of the environment in the coupling equations. In addition, the proposed algorithm poses a frequency switching mechanism that allows collective synchronization to cover a wide frequency spectrum. This multifrequency-coupled behavior names the multifrequency-coupled oscillators (MuFCO) algorithm.

This paper is organized as follows. The environment approach and the definition of the multiagent system are presented in Section II. The MuFCO algorithm is defined in Section III, where a variation of the Kuramoto model and a frequency switching mechanism are presented. In Section IV, an operation example of MuFCO algorithm in a real environment is shown. This is an example of how this algorithm can be used to coordinate consumptions in smart grids. Finally, the paper is concluded in Section V.

\section{ENVIRONMENT AND AGENTS}

In general, an environment may be limited depending on the problem studied. For example, an environment could be an electrical grid where the agents are the different connected loads. The relationship between this environment and these agents may be represented through different electrical variables. In the sake of simplicity, let the state of the environment be measured by a single-state variable represented by the signal $s(t)$. Returning to the example of the electrical grid, this state variable may be the consumed power in the grid, so all interaction between agents and environment may be represented in terms of power consumed, neglecting other possible variables. At the same time, the environment proposed in this paper is divided in two different parts.

1) An uncontrollable part that represents any event in the environment.

2) A controllable part that represents the multiagent system.

Both parts are represented by timeline signals in Fig. 1. The uncontrollable part $z(t)$ is defined as a single signal which is not affected by modifications in the environment, and its dynamics is unknown. On the other hand, the controllable part $p(t)$ is divided in different agents $x_{i}(t)$. Therefore, the environment $s(t)$ is defined as the sum of both parts as a sum of those signals

$$
s(t)=z(t)+p(t)
$$

The goal of the proposed algorithm is to control $p(t)$ to modify $s(t)$ by observing the same $s(t)$. At the same time, $p(t)$ is composed of a $M$ number of agents. The action of each agent is represented by $x_{i}(t)$, where $i$ is the agent identifier. The state of the controllable part is the sum of the actions of all agents, such that

$$
p(t)=\sum_{i=1}^{M} x_{i}(t) .
$$

The algorithm works in a distributed way by controlling each agent independently.

In this paper, the action of these agents is continuous, such that an agent may only modify the intensity of its action. In addition, the agents behave periodically, and they are

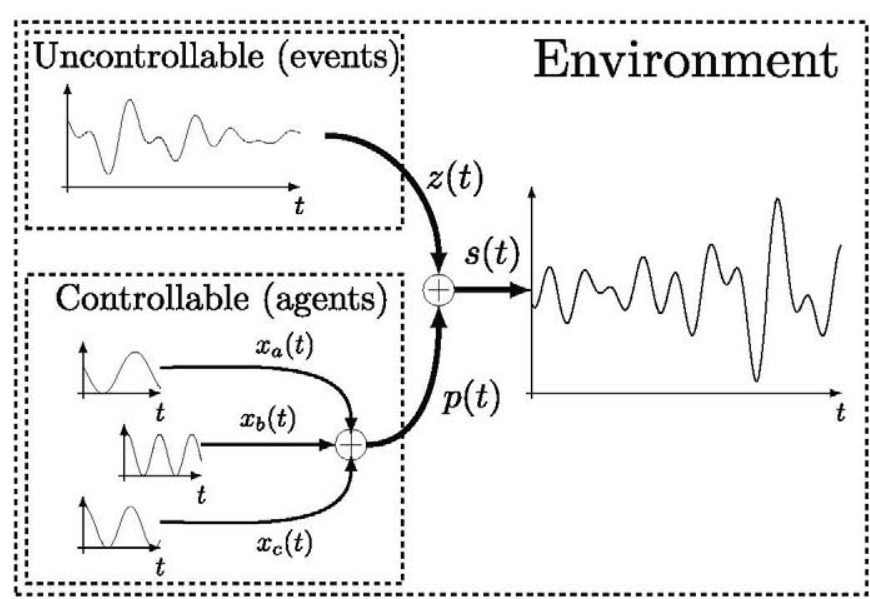

Fig. 1. Scheme of the proposed environment: $s(t)$ is the state signal of the environment; $z(t)$ is the state signal of the uncontrollable part that represents any event in the environment; $p(t)$ is the state signal of the controllable part that represents the agents; and $x_{a}(t), x_{b}(t)$, and $x_{c}(t)$ are the actions of three possible agents.

oscillators. The action of each agent is modeled as sinusoidal functions because of two main reasons.

1) They are the simplest periodic functions.

2) Any periodic signal can be implemented as a combination of sinusoidal functions.

This decision allows composing different periodic actions of the whole multiagent system $(p(t))$ as the composition of actions of different agents. Therefore, the action of an agent is defined by the following equation:

$$
x_{i}(t)=\sin \left(\omega_{i} t+\phi_{i}\right)
$$

where $\omega_{i}$ is the natural frequency and $\phi_{i} \in[-\pi, \pi)$ is the phase difference relative to a reference. The behavior of an agent can be modified by changing $\omega_{i}$ and $\phi_{i}$. Equation (1) can be also written as

$$
s(t)=z(t)+\sum_{i=1}^{M} \sin \left(\omega_{i} t+\phi_{i}\right) .
$$

The uncontrollable signal $z(t)$ may be of any nature, so it can be represented as a Fourier series and an error term

$$
z(t)=\sum_{n=0}^{N} A_{n}^{z} \cdot \sin \left(n \omega_{0} t+\phi_{n}^{z}\right)+e^{z}(t)
$$

where $N \in \mathbb{N}$ is the number of components of the Fourier series, $\omega_{0}$ is the fundamental frequency, $A_{n}^{z}$ and $\phi_{n}^{z}$ are the amplitude and phase of the $n$ component of $z(t)$, respectively, and $e^{z}(t)$ is the error term of $z(t)$ because of the approximation of the signal by a finite number of sinusoidal functions.

In the same way, the environment signal can be expressed as a Fourier series plus an error term. $s(t)$ can be decomposed in series with the same number of components $N$ and the same fundamental frequency $\omega_{0}$, such that

$$
s(t)=\sum_{n=0}^{N} A_{n}^{s} \cdot \sin \left(n \omega_{0} t+\phi_{n}^{s}\right)+e^{s}(t)
$$

where $A_{n}^{s}$ and $\phi_{n}^{s}$ are the amplitude and phase of the component $n$ of $s(t)$, respectively, and $e^{s}(t)$ is the error term of $s(t)$. 
The natural frequency $\omega_{i}$ of the agents can take any positive real value. To bind $x_{i}(t)$ with $s(t)$ and $z(t)$, it is established that the natural frequencies are contained in the set of frequencies of the Fourier series components: $\omega_{i} \in\left\{\omega_{0}, 2 \omega_{0}, 3 \omega_{0}, \ldots, N \omega_{0}\right\}$. By combining (4)-(6) together with this approach, it leads to the following equation:

$$
\begin{gathered}
\sum_{n=0}^{N} A_{n}^{s} \cdot \sin \left(n \omega_{0} t+\phi_{n}^{s}\right)+e^{s}(t)=\sum_{i=1}^{M} \sin \left(l_{i} \omega_{0} t+\phi_{i}\right) \\
+\sum_{n=0}^{N} A_{n}^{z} \cdot \sin \left(n \omega_{0} t+\phi_{n}^{z}\right)+e^{z}(t)
\end{gathered}
$$

where $l_{i} \in\{1, \ldots, N\}$ defines the natural frequency of the agent $i$ in the Fourier series. Notice that the error terms are equal through this approach $e^{s}(t)=e^{z}(t)$. Therefore, the relationship between $s(t), z(t)$, and $x_{i}(t)$ is reduced to a relationship between components of the Fourier series.

The agents can be grouped depending on their natural frequencies. All agents with same natural frequency, such that $l_{i}=n$, form a group of agents, where the number of agents of this group is denoted by $M_{n}$. By following this procedure and using the Euler's formula, (7) can be represented as

$$
\begin{aligned}
A_{0}^{s}+\sum_{n=1}^{N} e^{i n \omega_{0} t} e^{i \phi_{n}^{s}}= & A_{0}^{z}+\sum_{n=1}^{N} e^{i n \omega_{0} t} e^{i \phi_{n}^{z}} \\
& +\sum_{n=1}^{N} \sum_{j=1}^{M_{n}} e^{i n \omega_{0} t} e^{i \phi_{n, j}}
\end{aligned}
$$

where $\phi_{n, j}$ is the phase difference of the agent $j$ with the natural frequency $n \omega_{0}$. Notice that a group of agents could be empty so $M_{n}=0$. In this case, the summation of the actions of the agents is zero for this group.

This representation allows to split the relationship of (8) in each group of agents, such that

$$
A_{n}^{s} e^{i \phi_{n}^{s}}=A_{n}^{z} e^{i \phi_{n}^{z}}+\sum_{j=1}^{M_{n}} e^{i \phi_{n, j}} .
$$

The relationship between the uncontrollable signal $z(t)$ and the actions of the agents $x_{n, j}(t)$ can be assessed by an order parameter. This parameter is a macroscopic quantity that can be interpreted as the collective rhythm produced by oscillators [14], [15]. It is usually used only with a collective of coupled oscillators. Thanks to the Fourier series representation, $z(t)$ can be introduced in the order parameter. Let $r_{n} e^{i \Phi_{n}}$ be the order parameter of the component $n$, such that

$$
r_{n} e^{i \Phi_{n}}=\frac{1}{M_{n}+A_{n}^{z}}\left(A_{n}^{z} e^{i \phi_{n}^{z}}+\sum_{j=1}^{M_{n}} e^{i \phi_{n, j}}\right)
$$

where $r_{n}$ is the coherence of the component $n$ and $\Phi_{n}$ is the average phase. The synchronization of $z(t)$ and $x_{n, j}(t)$ can be assessed by $r_{n}$ which is in the range $[0,1]$. The order parameters are related with the amplitudes and phases of the $s(t)$ components. By combining (9) and (10)

$$
r_{n}=\frac{A_{n}^{s}}{M_{n}+A_{n}^{z}}, \quad \Phi_{n}=\phi_{n}^{s} .
$$

This relationship means that the order parameter may be calculated through the observation of the environment for each frequency component. In turn, the coherence between the uncontrollable signal and the agents may be known, allowing to perform a coupling process. For example, if $A_{n}^{s} \rightarrow 0$, then $r_{n} \rightarrow 0$ and $z(t)$ and $x_{n, j}(t)$ are in an incoherent state. On the other hand, if $A_{n}^{s} \rightarrow M_{n}+A_{n}^{z}$, then $r_{n} \rightarrow 1$ and $z(t)$ and $x_{n, j}(t)$ are fully synchronized.

Thanks to the Fourier series representation, the signals that compose the environment can be easily studied in the frequency domain through a Fourier analysis. However, to apply this approach to a real application with digital systems, the frequency analysis should be performed by a discrete Fourier transform (DFT). It means that the signal must be sampled at a certain period called sample period ( $\left.T^{\mathrm{smp}}\right)$. The number of samples of $s(t)$ considered in the frequency analysis is the processing window that is denoted by $W$. The use of the DFT implies that the information obtained from the frequency components of $s(t)$ is not the same as if working in continuous time. This calculus error must be taken into account, and both $T^{\text {smp }}$ and $W$ are parameters to be tuned when the proposed algorithm is applied to a concrete use.

\section{Multifrequency-COUPLEd OScillators}

\section{A. Coupling Equations}

In this section, the collective synchronization of a group of agents with a single-frequency component is studied. It means that the uncontrollable signal $z(t)$ is reduced to a single sinusoidal function and all agents work on this natural frequency. Hence, the environment signal is also a sinusoidal function whose amplitude and phase depend on the amplitude of $z(t)$, the number of agents, and the phase relationship. The subscript $n$, which indicates the component of the Fourier series, has been removed from the equations because the procedure followed in this section is valid for any component. In this case, only the phase differences of the agents can be modified because the natural frequencies are fixed. This phase difference modifications are based on the Kuramoto model [15].

The Kuramoto model defines the coupling between a collective of oscillators which represent the action of the agents in this paper. The uncontrollable signal is also included in the coupling process. It is simplified to a single sinusoidal function $z(t)$ and, under this assumption, it can be considered as an uncoupled oscillator. Although $z(t)$ is a passive element, it affects the coupling process through the order parametersee (10). Therefore, the phase differences of $x_{i}(t)$ vary in time $\phi_{i} \rightarrow \phi_{i}(t)$ but the phase difference of $z(t)$ keeps constant $\phi^{z}=$ const. The Kuramoto model applied to the phase differences leads the following coupling equation:

$$
\dot{\phi}_{i}(t)=K \cdot r(t) \cdot \sin \left(\Phi(t)-\phi_{i}(t)\right)
$$

where $K \in \mathbb{R}$ is the coupling strength. $K$ defines the coupling process from a qualitative and quantitative point of view. The absolute value of $K$ affects the coupling velocity which in turn affects the dynamical behavior of the coupling process. The sign of $K$ defines qualitatively the interaction between the 


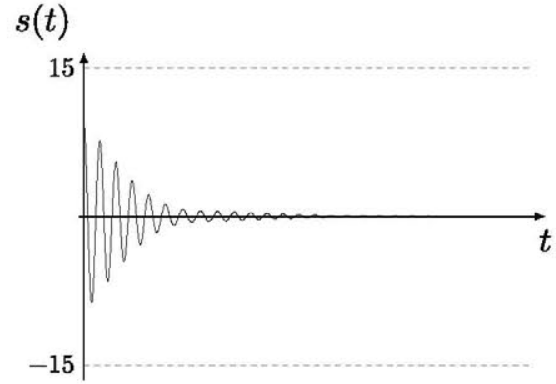

(a)

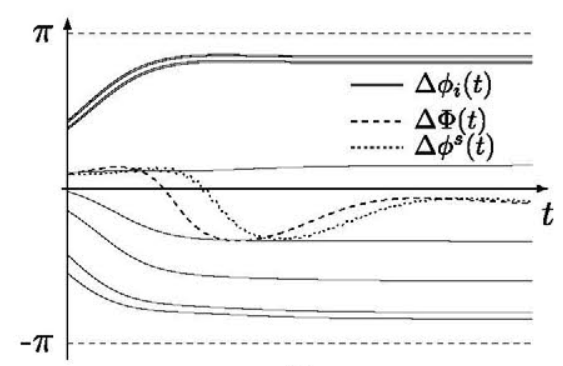

(b)

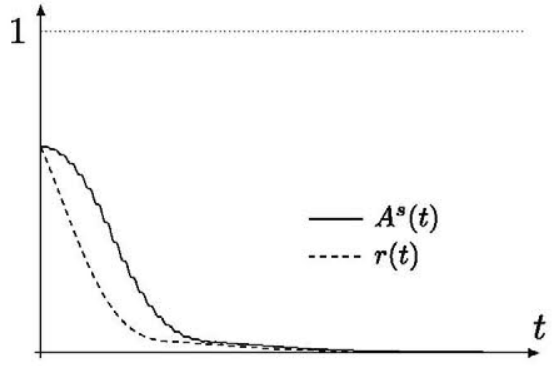

(c)

Fig. 2. Example of negative coupling between agents and $z(t)$ using (13) with $K=-0.01, M=10, \omega^{z}=\omega_{i}={ }^{2 \pi} / 32 \mathrm{rad} / \mathrm{s} \forall i, A^{z}=5, W=$ 64 samples, and $T^{\mathrm{smp}}=1 \mathrm{~s}$ : (a) $s(t)$; (b) $\phi_{i}(t), \Phi(t)$ and $\phi^{s}(t)$; and (c) $r(t)$ and normalized $A^{s}(t)$.

agents and $z(t)$. Positive $K$ values correspond to an attractive interaction. It implies that agent phases tend to the $z(t)$ phase being fully synchronized. On the other hand, negative $K$ values produce a repulsive interaction. Agent phases and $z(t)$ phase tend to separate each other, reaching an incoherent state where $A^{s} \rightarrow 0$. Along this paper, the agents are used to remove the frequency components of $s(t)$ as a bank of filters. For this reason, $K$ is always negative.

The use of the order parameter to perform (12) implies that the phases of all agents should be known instantaneously. Depending on the situation, this requirement may hinder the application of collective synchronization on distributed systems. This paper proposes to approximate the order parameter by the DFT of $s(t)$ by using (11). Through this approach, every agent may obtain the required coupling information only by observing the environment signal $s(t)$. Although the DFT allows the coupling process without the knowledge of agent phases, the coupling process still requires $M$ and $A^{z}$ to be implemented. The proposed algorithm aims to adapt to unknown environments and populations. Hence, the normalization of $A^{s}$ by $M+A^{z}$ should be modified by another normalization factor which does not require the knowledge of $M$ and $A^{z}$. For this reason, $M+A^{z}$ is replaced by the historical maximum of the frequency component of the environment signal $\max \left(A^{s}\right)$. The reason of this proposal is that the maximum value attainable by a component of $s(t)$ is $M+A^{z}$. Combining (11) and (12) together with this approximation leads the following coupling equation:

$$
\dot{\phi}_{i}(t)=K \cdot \frac{A^{s}(t)}{\max \left(A^{s}\right)} \cdot \sin \left(\phi^{s}(t)-\phi_{i}(t)\right) .
$$

Fig. 2 shows an example of negative coupling between agents and $z(t)$ using (13) with $K=-0.01$. The frequency of the uncontrollable signal $z(t)$ is $\omega^{z}=2 \pi / 32 \mathrm{rad} / \mathrm{s}$ and the natural frequencies of all agents are $\omega_{i}=2 \pi / 32 \mathrm{rad} / \mathrm{s} \forall i$. The amplitude of $z(t)$ is $A^{z}=5$. The number of agents is $M=10$. Thus, if the agents and $z(t)$ are in an incoherent state, the amplitude of $s(t)$ takes the value $A^{s}=0$. The phase of $z(t)$ is the phase reference; thus, $\phi^{z}=0$ and the phase difference of the agents $\phi_{i}(t)$ are referenced to this phase. The initial phase differences of the agents are uniformly distributed in $[-\pi, \pi)$. Fig. 2(a) shows how $s(t)$ develops in time. $s(t)$ begins with an amplitude $A^{s} \approx 10$ but converges to an incoherent state with
$A^{s}=0$. In this case, the phase differences converge to an incoherent state-see Fig. 2(b). It implies that they are spread in the range $[-\pi, \pi)$ such that the phase coherence is reduced to zero. Fig. 2(c) shows the development in time of $r(t)$. The use of the DFT to calculate $A^{s}(t)$ and $\phi^{s}(t)$ and to replace $r(t)$ and $\Phi(t)$ allows to perform a collective synchronization without calculating the order parameter. In addition, the agents achieve a incoherent state despite using the $\max \left(A^{s}\right)$ approximation.

The DFT has been applied to $s(t)$ using a sample period of $T^{\mathrm{smp}}=1 \mathrm{~s}$, and the processing window is $W=64$ samples. The DFT gives the amplitude $A^{s}(t)$ and phase difference $\phi^{s}(t)$ of the frequency component analyzed in this example $\omega_{0}=2 \pi / 32 \mathrm{rad} / \mathrm{s}$. Fig. 2(b) shows the development of $\phi^{s}(t)$ calculated by the DFT over the example. According to (11), $\phi^{s}(t)$ and $\Phi(t)$ should be equal, but the information obtained from the Fourier analysis is not exact because of the use of a DFT. In general, $W$ affects to this result by causing a certain delay between the instantaneous phase $\Phi(t)$ and the phase calculated through the DFT $\phi^{s}(t)$. Fig. 2(c) shows the development of the normalized $A^{s}(t)$ calculated by the DFT over the example. $A^{s}(t)$ has been normalized by $A^{z}+M$ to restrict it in the range $[0,1]$ and to be compared with the phase coherence. According to (11), normalized $A^{s}(t)$ should be equal to $r(t)$, but a certain delay can be observed as in the case of the phase differences. Although this detail, synchronization is achieved using a DFT instead of a full knowledge of the phases of the oscillators. This procedure has a practical advantage in the use of collective synchronization in distributed multiagent systems.

\section{B. Multifrequency Coupling}

The previous coupling equations consider that all agents and $z(t)$ have the same frequency. However, the uncontrollable signal and consequently the environment rarely are a sinusoidal function. It is commonly formed by several frequency components or even stochastic signals. The modification of the natural frequencies of the agents is required to adapt them to complex environments. The natural frequency modification mechanism, also called frequency switching, is described in this section. This mechanism allows the multiagent system works on different natural frequencies, where each agent is coupled to a certain frequency component through (13). This multifrequency-coupled behavior names the MuFCO algorithm. 


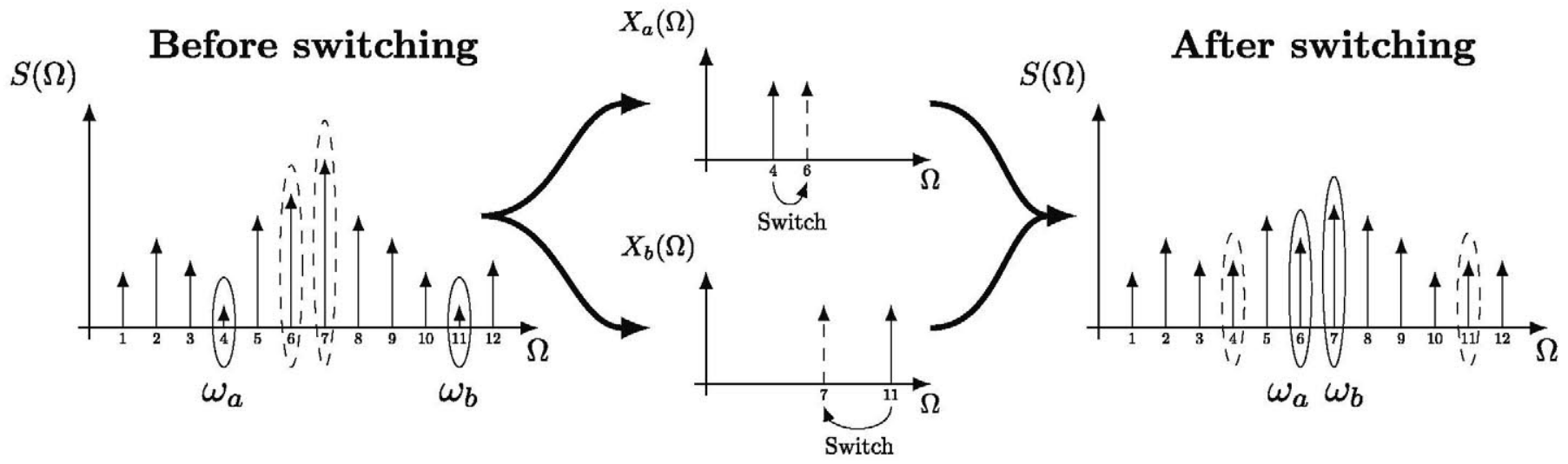

Fig. 3. Conceptual example of the MuFCO algorithm operation with two agents: $a$ and $b$. Before switching, the natural frequencies are $\omega_{a}=4 \omega_{0}$ and $\omega_{b}=11 \omega_{0}$. The goal of the MuFCO algorithm in this example is to reduce the main frequency components of $s(t)$. Thus, the natural frequencies of the agents will switch to the most prominent components of $S(\Omega)$. The coupling strength is negative in this example. After switching, the natural frequencies are $\omega_{a}=6 \omega_{0}$ and $\omega_{b}=7 \omega_{0}$. This mechanism reduces the amplitudes of $A_{6}^{s}$ and $A_{7}^{s}$. On the other hand, $A_{4}^{s}$ and $A_{11}^{s}$ increase because of the absence of these agents.

Let $S(\Omega)$ be the DFT of $s(t)$. The goal of MuFCO algorithm is to achieve a target shape of $S(\Omega)$. This target shape defines the coordination process by indicating the desired frequency characteristics of the environment. Fig. 3 shows a conceptual example of the MuFCO algorithm operation. In this example, the objective is to smooth $s(t)$ by removing the most prominent frequency components. Thus, the target shape of $S(\Omega)$ is zero for every frequency component. It means that the agents behave like a bank of filters. The coupling strength is paramount to achieve the target shape. In this case, a negative coupling is required. First, $S(\Omega)$ has a certain shape because of $z(t)$ and the initial $\omega_{i}$ of every agent. In this example, there are two agents: $a$ and $b$. Before switching, $\omega_{a}$ is in the fourth component of $S(\Omega)$ and $\omega_{b}$ is in the eleventh component. In order to reduce the frequency components with the highest amplitude, the agent $a$ switches from the fourth component to the sixth and the agent $b$ switches from the eleventh component to the seventh. The amplitudes of these frequency components are reduced because of the negative coupling. On the other hand, $A_{4}^{s}$ and $A_{11}^{s}$ increase because of the absence of these agents. With a sufficient number of agents, all components may be reduced.

MuFCO algorithm is a distributed algorithm which implies that each agent chooses its natural frequency. The frequency switching is divided in two steps: 1) the switching decision, where each agent decides to stay in the current frequency component or switch; and 2) the new frequency component selection, where each agent chooses its new natural frequency. The switching decision is done through the calculation of the shape error function $E(S(\Omega))$. It evaluates the difference between the target shape and the current shape of $S(\Omega)$. The output range of $E(S(\Omega))$ is [0,1], where 0 denotes that both shapes are equal and 1 denotes the maximum difference to be evaluated. $E(S(\Omega))$ is used as the stop condition so that the lower its value, the lower the probability that an agent switches to another frequency component. If an agent decides to switch to another frequency component, it uses the transition rule $T(S(\Omega), n)$. The transition rule generates a probability density function from the spectrum of $s(t)$, where $T(S(\Omega), n)$

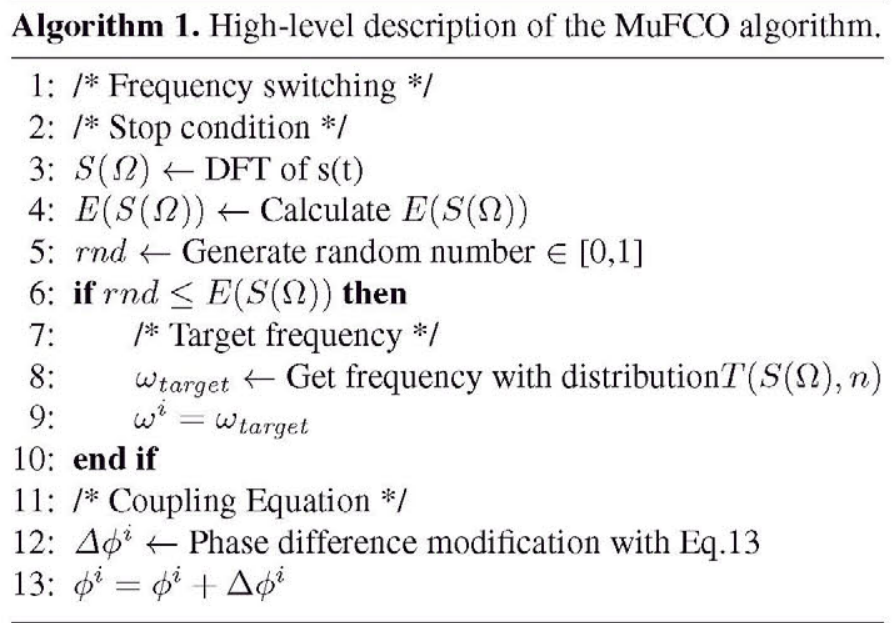

is the probability to switch to frequency component $n$. Each agent chooses its new natural frequency following this density function.

Algorithm 1 describes the operation of the MuFCO algorithm performed by every agent individually. It is executed each new sample, it means every $T^{\text {smp }}$. First, the agent decides whether switch or not its frequency using the shape error function-see from line 3 to 6 . Second, if the agent switches, it chooses its new natural frequency with the transition rule-see from line 8 to 9 . Finally, this agent modifies its phase using (13) - see from line 12 to 13 . Once the frequency and phase have been modified, the action of the agent is modified by following (3).

Fig. 4 shows an example of the MuFCO algorithm operation. There is an uncontrollable signal $z(t)$ which is composed by three frequency components: 1) $\omega_{5}^{z}=2 \pi^{5} / 64 \mathrm{rad} / \mathrm{s}$; 2) $\omega_{15}^{z}=2 \pi^{15} / 64 \mathrm{rad} / s$; and 3) $\omega_{25}^{z}=2 \pi^{25} / 64 \mathrm{rad} / s$. The amplitudes of these frequency components are $A_{5}^{z}=50, A_{15}^{z}=30$, and $A_{25}^{z}=20$. The DFT of $s(t)$ is calculated with $T^{\text {smp }}=1 \mathrm{~s}$ and $W=64$ samples. Fig. 4(a) shows the DFT of $z(t)$. There are 100 agents with $K=-0.01$. The initial natural frequencies of the agents are uniformly distributed over the spectrum. In addition, the initial phase differences are uniformly distributed 


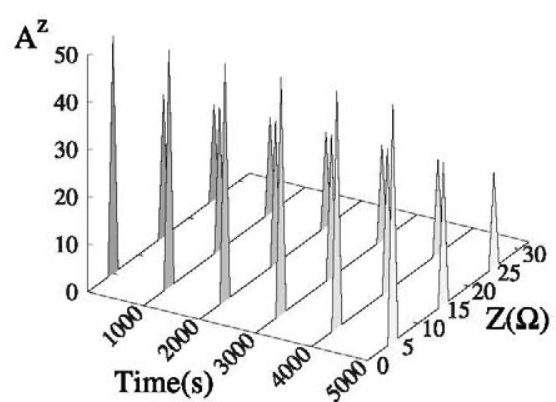

(a)

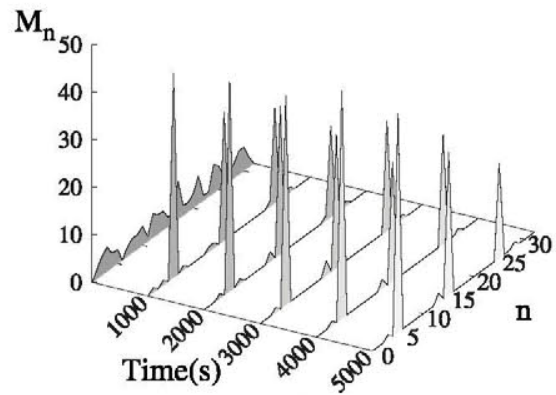

(b)

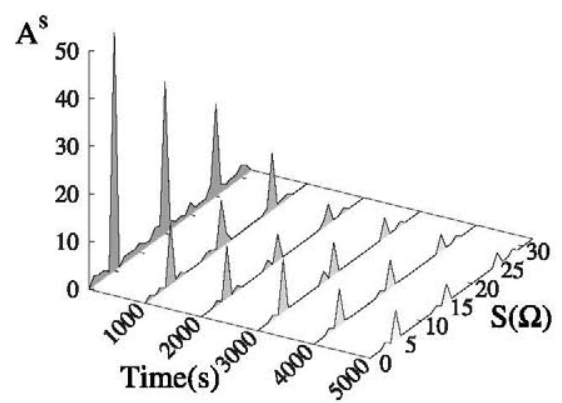

(c)

Fig. 4. Representation in the frequency domain of the proposed example of the MuFCO algorithm operation. There are 100 agents with $K=-0.01$. The uncontrollable signal has the following frequency components: $A_{5}^{z}=50, A_{15}^{z}=30$, and $A_{25}^{z}=20$. The DFT is calculated with $T^{\text {smp }}=1$ s and $W=64$ samples. This example lasts for $5000 \mathrm{~s}$. (a) Development in time of the DFT of $z(t)$. (b) Distribution of natural frequencies of $x_{i}(t)$ during the example. (c) Development in time of the DFT of $s(t)$.

in $[-\pi, \pi)$. Fig. 4(b) shows the natural frequencies distribution of the agents. The DFT of $s(t)$ is shown in Fig. 4(c).

The following equation defines the shape error function for this example:

$$
E(S(\Omega))=\frac{P_{\text {switch }}}{W / 2} \cdot \sum_{j=1}^{W / 2}\left(\frac{A_{j}^{s}}{\max (S(\Omega))}\right)^{2}
$$

where $P_{\text {switch }}$ is the switching factor that denotes the probability that an agent switches to another frequency component, and $\max (S(\Omega))$ is the historical maximum amplitude of any frequency component measured during the example execution.

The transition rule for this example is defined by the following equation:

$$
T(S(\Omega), n)=\frac{\left(A_{n}^{s}\right)^{2}}{\sum_{j=1}^{W / 2}\left(A_{j}^{s}\right)^{2}} .
$$

In this case, the higher the value of $A_{n}^{s}$, the higher the probability to switch to the frequency component $n$.

In this example, MuFCO algorithm is executed during 5000 s. $Z(\Omega)$ keeps constant because this signal has been defined as a fixed number of sinusoidal functions-see Fig. 4(a). On the other hand, the agents switch their natural frequencies, and they couple to their respective frequency components. They switch to the frequency components with the higher amplitudes of $S(\Omega)$. For this reason, their natural frequencies end up being the same that the initial main components of $S(\Omega)$-compare Fig. 4(b) with Fig. 4(c). Thanks to the negative coupling, these components are removed. The development of $S(\Omega)$ in time shows how $A_{5}^{s}, A_{15}^{s}$, and $A_{25}^{s}$ are decreased until they achieve a value close to zero.

\section{Operation Example: Smart Grid Application}

MuFCO algorithm may be used in different applications where the environment and the actions of the agents can be modeled as signals. In this section, the MuFCO algorithm is used to coordinate consumptions in a smart grid. In general, the consumption of an electrical grid behaves periodically: the consumption profile almost repeats every day, week, or season.
The management of the consumption of these grids is receiving increasing attention by research and industry with the so called demand-side management (DSM) [16]. One of the main objectives of the DSM is the smooth of the aggregated consumption by reducing the difference between maximum and minimum consumption power [17]. This smoothing requires the coordination of thousand or even million of elements spread over a certain area. The coordination of these elements to use properly the available resource becomes a complex task which is addressed by the so-called smart grids.

DSM in smart grids enhances its capabilities regarding the classical electrical grids. The convergence of information and communications technologies (ICTs) with power system engineering allows new levels of automation. DSM in this context is usually formulated as optimization problems, which may be solved by various approaches [18]. For example, [19] presents a residential load control based on convex optimization which achieves an equilibrium between economical user benefit and social welfare. In [20], game theory is used to solve an energy consumption scheduling game to minimize energy costs. In [21], self-organizing agents are able to solve fundamental control and monitoring problems in smart microgrids. These approaches usually require dynamic pricing to coordinate the users' facilities with the utility company. In this paper, a new approach based on the use of collective synchronization of a multiagent system is proposed. MuFCO algorithm coordinates different agents to smooth the consumption of the smart grids in a self-organized way, where self-organized means that each agent defines a consumption profile without communicating with other. Thus, the only information received by the agents is the aggregated consumption. One of the main differences from previous works is that MuFCO algorithm does not use pricing information.

For this operation example, the electrical grid is defined as a single node disregarding losses and transmission delays. The electrical grid contains facilities of different users which contain in turn different electrical loads. The consumption in time of the facility $i$ is represented as the power signal $p_{i}(t)$. The sum of all facilities in the electrical grid is the aggregated consumption such that $s(t)=\sum_{i=1}^{M} p_{i}(t)$, where $M$ is the number of facilities in the electrical grid. In this example, the 


\section{Without MuFCO}

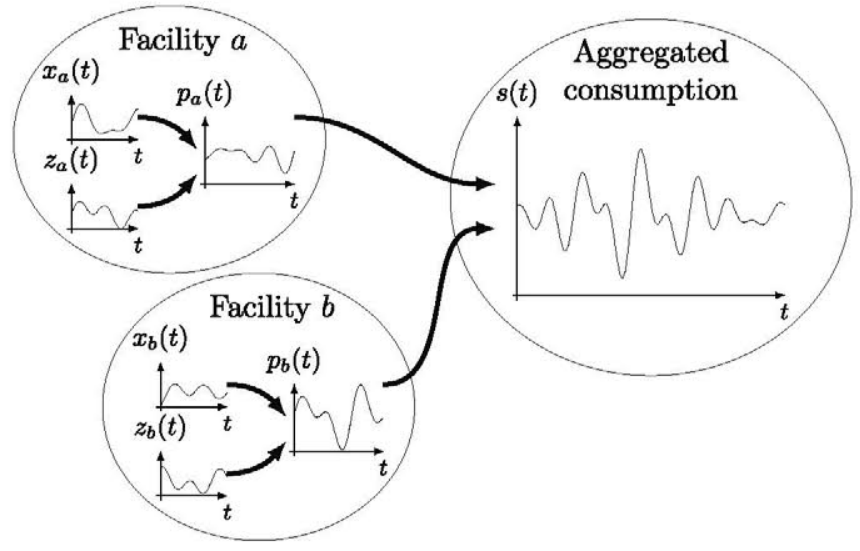

With MuFCO

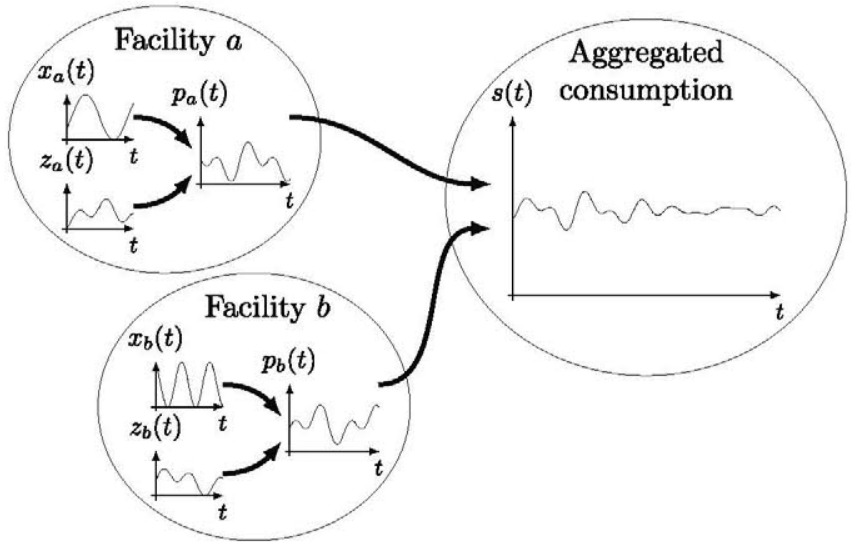

Fig. 5. Schematic example of the proposed electrical grid where there are two facilities $a$ and $b . p_{a}(t)$ and $p_{b}(t)$ represent the consumed power of each facility. $x_{a}(t)$ and $x_{b}(t)$ are the controllable consumptions which are managed by the MuFCO algorithm. $z_{a}(t)$ and $z_{b}(t)$ are the uncontrollable consumptions of facilities $a$ and $b$, respectively. $s(t)$ is the aggregated consumption of the electrical grid. This example is divided in two parts: 1 ) without MuFCO where the controllable consumption is not synchronized; and 2) with MuFCO where the controllable consumption is synchronized with the grid.

aggregated consumption is the environment signal. Each facility is divided into a controllable part and an uncontrollable part. The uncontrollable part represents all consumptions that cannot be managed by the MuFCO algorithm because of their energy requirements or simply because control technology is not available. The uncontrollable consumption of the facility $i$ is represented by $z_{i}(t)$. The controllable part represents consumption that can be managed by the MuFCO algorithm, e.g., water pumps, HVAC, ${ }^{1}$ and electric vehicle charging. The controllable consumption of the facility $i$ is represented by $x_{i}(t)$, which is the action of the agent $i$. The action of the agents $x_{i}(t)$ is used as consumption patterns. If an agent controls the consumption of a electrical system, the power of this system will be modified such that its consumption will shape $x_{i}(t)$. In this paper, an ideal case is considered, where the consumptions controlled by the MuFCO algorithm can shape sinusoidal functions. Fig. 5 shows a schematic example of the proposed electrical grid. This representation is consistent with the definition of the environment in Section II. Notice that, although each uncontrollable consumption $z_{i}(t)$ belongs to a single facility, the sum of them can be considered as a single uncontrollable consumption of the whole grid $z(t)$.

The electrical grid chosen for this example is the peninsular Spanish grid during the year 2014. Fig. 6(a) shows the aggregated consumption during this year. This consumption varies along the year with a yearly average consumption of $28.1 \mathrm{GW}$, a maximum consumption around $39.5 \mathrm{GW}$, and minimum consumption around $17.2 \mathrm{GW}$. The daily difference between peak and valley has also been calculated so that the maximum difference throughout the year is $15.5 \mathrm{GW}$, the minimum is $7.6 \mathrm{GW}$, and the average is $11.7 \mathrm{GW}$. Fig. 6 (b) zooms on a signal segment showing the consumption of the electrical grid during 2 weeks. The intraday variation can be appreciated in this figure. This variation is the strongest in the consumption signal. The spectrum of the aggregated consumption is shown in Fig. 6(c).
The strongest frequency component has a period of $24 \mathrm{~h}$ verifying the importance of intraday variation. The next strongest component is in the 12-h period. For periods longer than $24 \mathrm{~h}$, the main frequency component corresponds to the weekly variations with a 168 -h period. In this case, MuFCO algorithm is not enabled; thus, the consumption of the electrical grid is directly the environment and the uncontrollable signal $s(t)=z(t)$.

In order to analyze the variability of the aggregated consumption, the Crest Factor $C$ has been used. It is a measure of a waveform, showing the ratio of peak values to the average value, such that

$$
C=\frac{|s|^{\text {peak }}}{s^{\text {rms }}} ; \quad s^{\text {rms }}=\sqrt{\frac{s_{1}^{2}+s_{2}^{2}+\cdots+s_{N}^{2}}{N}}
$$

where $N$ is the number of samples taken from the aggregated consumption, $|s|^{\text {peak }}$ is the absolute value of the maximum peak, and $s^{\text {rms }}$ is the root-mean square. The crest factor makes reference to a concrete time interval in which the aggregated consumption is evaluated. The time interval is denoted with a subscript, e.g., the crest factor of a day is denoted by $C_{\text {day }}$. In this analysis, $C_{\text {year }}, C_{\text {month }}, C_{\text {week }}$, and $C_{\text {day }}$ have been calculated. These crest factors are averaged during the whole year, where $\bar{C}_{\text {day }}$ represents the average of the crest factors of the 365 days, $\bar{C}_{\text {week }}$ is the average of the 52 weeks, and $\bar{C}_{\text {month }}$ is the average of the 12 months. Table I, "without MuFCO" column, shows the crest factor averages for the aggregated consumption exposed in Fig. 6.

The example shown in this section is performed with 200 agents of $100 \mathrm{MW}$ of peak power: MuFCO algorithm is controlling $20 \mathrm{GW}$ of the electrical grid $(\approx 50 \%$ of the yearly maximum peak). Recall that an agent ideally controls an electrical system of any size. The electrical grid is defined by the following equation:

$$
s(t)=z(t)+\sum_{i=1}^{200} 100 \mathrm{MW} \sin \left(\omega_{i} t+\phi_{i}\right)
$$




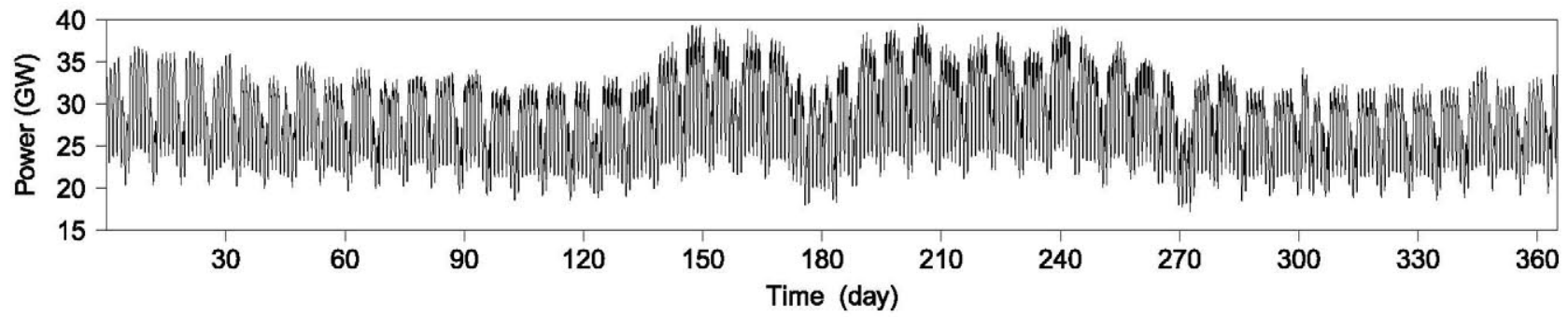

(a)

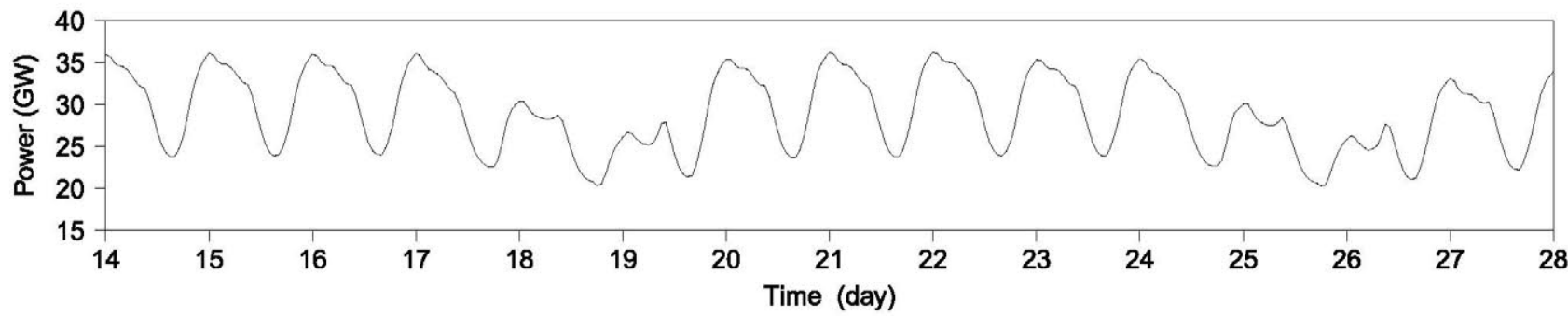

(b)

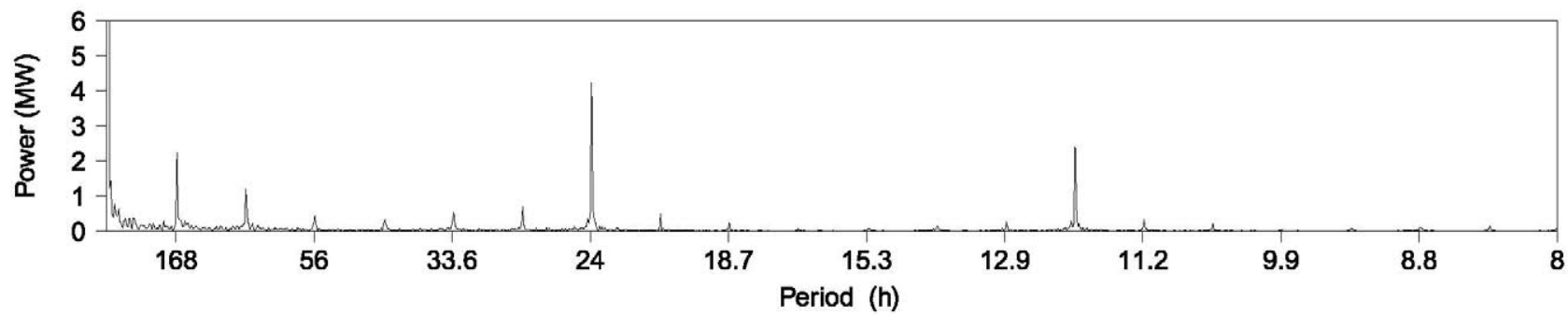

(c)

Fig. 6. Aggregated consumption of peninsular Spain during 2014: (a) consumption of the electrical grid in time $(s(t)=z(t))$; (b) zoom on 2 weeks of $s(t)$; (c) DFT of the aggregated consumption. Source: Spanish grid operator (R.E.E.)

TABLE I

Crest factors of the AggRegated Consumption

\begin{tabular}{ccl}
\hline Crest factors & Without MuFCO & $\mathcal{C}_{\text {day }}$ \\
\hline$C_{\text {year }}$ & 1.3893 & 1.2843 \\
$\bar{C}_{\text {month }}$ & 1.2856 & 1.1772 \\
$\bar{C}_{\text {week }}$ & 1.2411 & 1.1340 \\
$\bar{C}_{\text {day }}$ & 1.1657 & 1.0751 \\
\hline
\end{tabular}

where $s(t)$ is the aggregated consumption of the electrical grid modified by the agents controlled by the MuFCO algorithm, $z(t)$ is the uncontrollable part of the grid that coincides with the aggregated consumption of the example of Fig. 6, and the sinusoidal functions are the consumption patterns of all agents. The MuFCO algorithm uses the shape error function of (14) and the transition rule of (15).

The MuFCO algorithm has four configurable parameters: 1) $K$;2) $P^{\text {switch }}$;) $T^{\text {smp }}$; and 4) $W$. Their values depend on the environment nature and optimization conditions. The optimization conditions refer to a concrete objective, it means how the $\mathrm{MuFCO}$ algorithm should modify the environment signal $s(t)$. In this example, the algorithm is tuned to reduce the intraday variations of the electrical grid. The tuning process can be done with different techniques of algorithm optimization as genetic algorithms, ACO, PSO, etc. In this paper, the MuFCO algorithm has been optimized using the iterated racing procedure [22]. Its main purpose is to automatically configure optimization algorithms by finding the most appropriate settings given a set of instances of an optimization problem. irace executes different instances of the problem with different configurable parameter combinations of the $\mathrm{MuFCO}$ algorithm. An instance of the problem represents a specific execution framework where the MuFCO algorithm operates with a certain combination of the configurable parameters. After the execution of an instance, the MuFCO algorithm performance must be assessed through a cost function. The goal of irace is to find the best combination of configurable parameters that minimizes the cost function for the different instances. In order to assess the intraday variations, the cost function is the average daily crest factor $\bar{C}_{\text {day }}$. The resulting optimization process shows that the value of the configurable parameters that reduces the intraday variations are: $K=-0.03, P^{\text {switch }}=0.02, T^{\mathrm{smp}}=90$, and $W=16$. It is noteworthy that the sample period is $90 \mathrm{~min}$; thus, the execution of the MuFCO algorithm only takes place in this interval. This means that low computing power is required.

Fig. 7 shows an example of the MuFCO algorithm operation optimized to reduce intraday variations. The aggregated consumption with $20 \mathrm{GW}$ controlled by the algorithm is shown in Fig. 7(a). The intraday variations of the aggregated consumption have been considerably reduced in comparison with the situation of Fig. 6(a). The yearly average consumption is $28.1 \mathrm{GW}$, the maximum consumption is $35.8 \mathrm{GW}$, and the minimum consumption is $17.9 \mathrm{GW}$. The maximum daily difference between peak and valley throughout the year is $11.2 \mathrm{GW}$, the minimum is $1.3 \mathrm{GW}$, and the average is $4.7 \mathrm{GW}$. This effect can be better appreciated by observing Fig. 7(b). The DFT of the aggregated consumption also shows the intraday variation reduction. Fig. 7(c) shows that the frequency components of 24and 12-h period have almost been removed in comparison with 

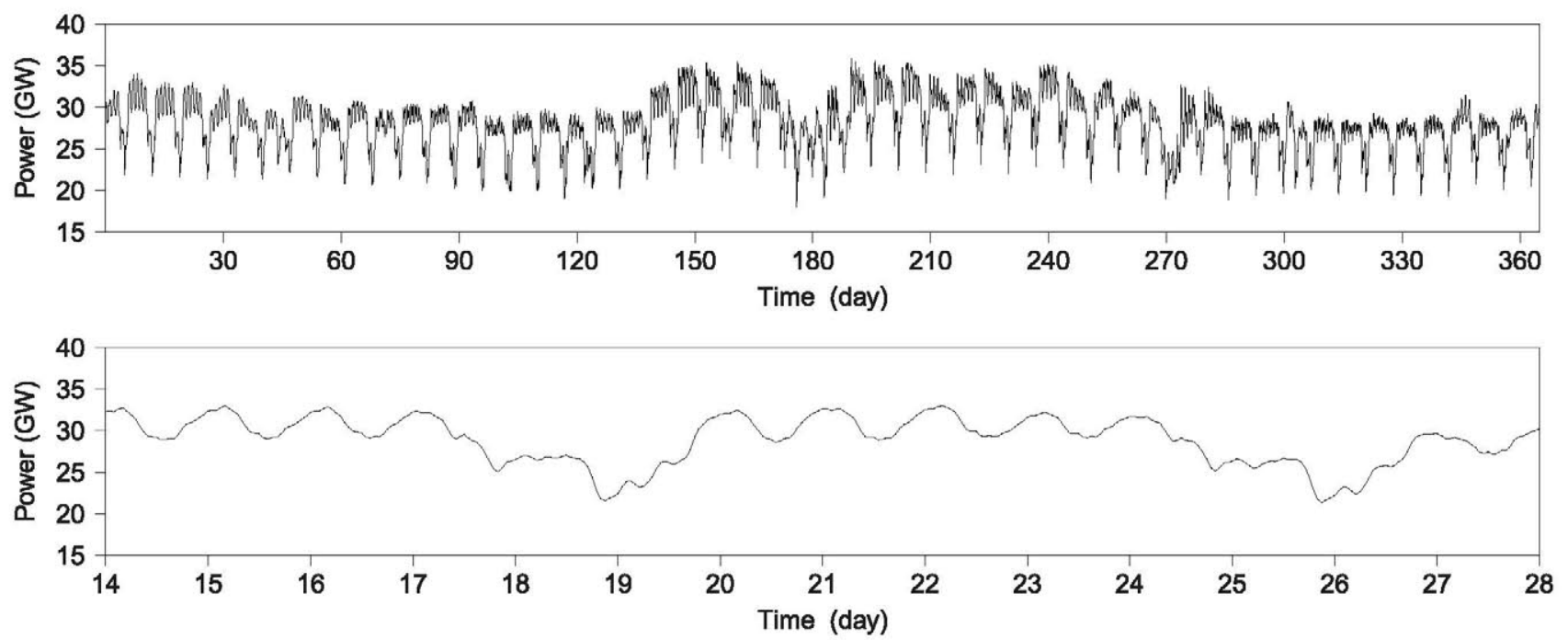

(b)

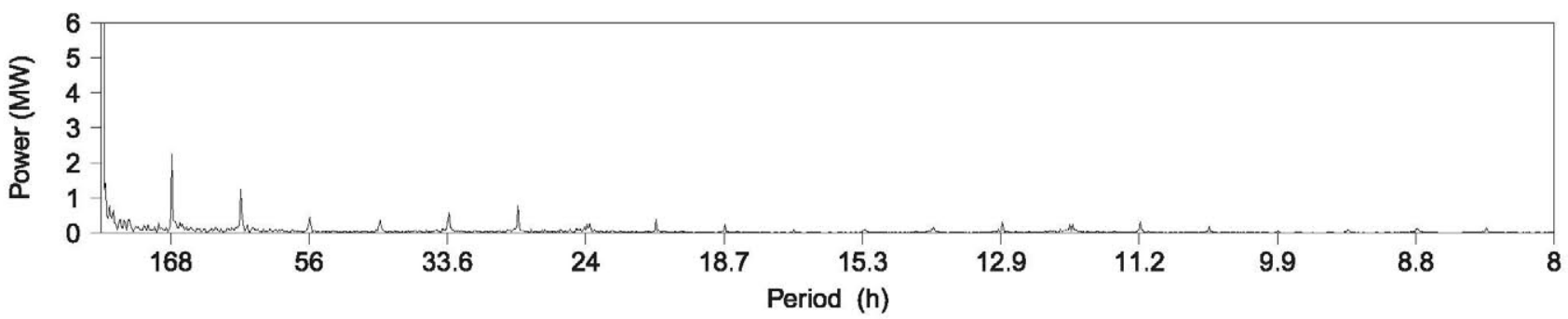

(c)

Fig. 7. Aggregated consumption of peninsular Spain during 2014 using MuFCO algorithm with daily optimization: (a) consumption of the electrical grid in time $\left(s(t)=z(t)+\sum_{200}^{i=1} x_{i}(t)\right)$; (b) zoom on 2 weeks of $s(t)$; (c) DFT of the aggregated consumption.

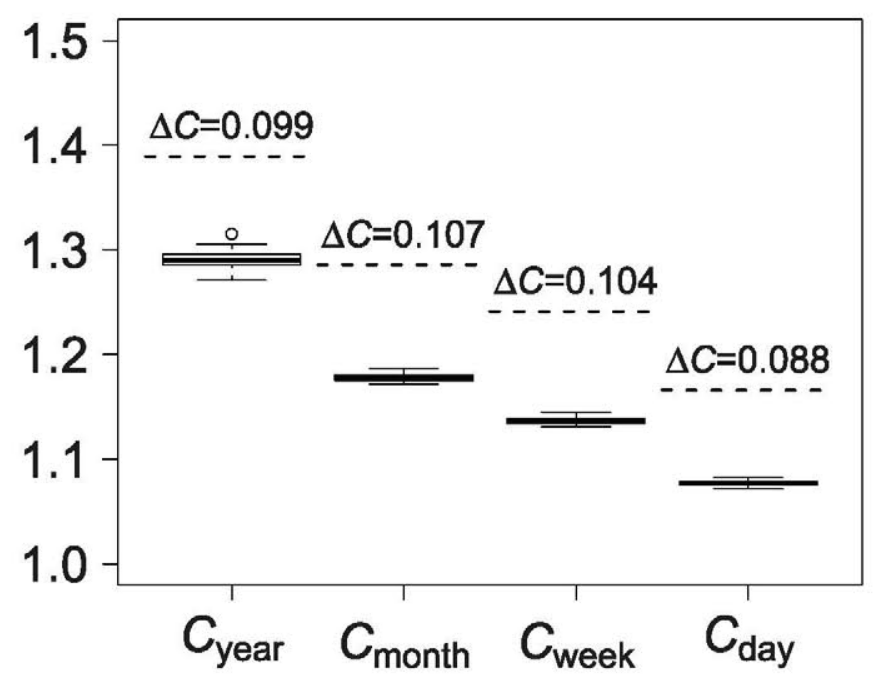

Fig. 8. Results of the statistical analysis of the example of the MuFCO algorithm operation to reduce the uncertainty introduced by the random component. These results are shown through a boxplot representation. Dashed lines represent the crest factors when there are not consumptions controlled by the MuFCO algorithm (results of the example of Fig. 6). $\Delta C$ is the difference between the crest factors without MuFCO and the mean of the crest factors obtained from the analysis. The crest factors have been reduced for all time intervals.

Fig. 6(c). The crest factor averages for this example are shown in Table I, $\mathcal{C}_{\text {day }}$ column. All crest factors have been reduced with special emphasis on the daily crest factor.

In the previous example, the reduction of the crest factors using MuFCO algorithm has been shown. In the following, a statistical analysis of this example is done to reduce the uncertainty introduced by the random component. To carry out this analysis, 100 experiments have been performed using MuFCO with the optimized parameters. These experiments have the same configuration as the previous example. They only differ in the seed of the random number generator. Fig. 8 shows the result of the statistical analysis through a boxplot representation. It shows the boxplot for the four types of crest factors. The crest factors of the aggregated consumption without $\mathrm{MuFCO}$ are also represented with discontinuous lines. They correspond to the example of Fig. 6, where there are no random components. These crest factors are used as reference of the improvement brought by the MuFCO algorithm. Thereby the crest factor improvement $\Delta C$ is also shown in the graphs. $\Delta C$ is the difference between the crest factors without $\mathrm{MuFCO}$ and the mean of the crest factors obtained from the analysis. All crest factors are reduced by using MuFCO algorithm with the optimized configurable parameters.

\section{CONCLUSION}

In this paper, an algorithm called MuFCO which implements a multiagent coordination in periodic environments has been proposed. The coordination has been addressed from the signal processing point of view, where all elements of the environment are signals. The agents behave as oscillators whose actions are sinusoidal functions. The environment signal has been divided in different frequency components. The agents 
are coupled to these frequency components by performing a coupling equation based on the Kuramoto model. Thanks to this approach, an uncontrollable signal could be introduced in the coordination process. In addition, the order parameter has been replaced by the information of the frequency components what allows a real-time implementation of the algorithm. The natural frequencies can take different values in a discrete range of these frequency components. The agents are coupled to the component in which its natural frequency is. This multifrequency-coupled behavior names the proposed MuFCO algorithm. With this algorithm, the multiagent system can coordinate and adapt in complex environments.

The coordination of multiagent systems with the environment from the signal processing point of view allows the collective synchronization to be applied to more practical applications. In this paper, the environment signals has been analyzed through a DFT which is a widely use technique in signal processing. However, the use of the DFT implies that the information obtained from the frequency components of the environment signal is not the same as in continuous time. The sampling period and the processing window of the DFT become key parameters for the MuFCO algorithm operation, and their proper value depends on the application. On the other hand, the introduction of an uncontrollable signal presents a new synchronization approach. The coordination can be performed from a distributed point of view, where no information is required from every agent. The agents do only require information from the environment through the DFT. In addition, the oscillators can modify their natural frequencies by performing a multifrequency behavior. This procedure differs from most of works on coupled oscillators where the natural frequencies have a certain distribution close to a central frequency. To perform this modification or frequency switching, a probability decision function has been defined which is called transition rule. Through this rule, every oscillator chooses its natural frequency in a certain discrete range. This algorithm opens up a new approach to the application of such techniques and to the adaptation of collectives of oscillators to complex environments.

$\mathrm{MuFCO}$ algorithm may be applied in different applications where the environment and the action of the agents can be modeled as signals. In Section IV, the MuFCO algorithm has been used to coordinate consumptions in a smart grid. Through this coordination, the consumption of an electrical grid is smoothed. In this example, the algorithm can coordinate the consumption of the multiagent system by executing every $90 \mathrm{~min}$. This is a sign of the low computational load which can be reached by MuFCO algorithm. In addition, the distributed approach makes not necessary a central coordinator and reduces the communication requirements: agents only need to receive the aggregated consumption signal. These features present some advantages for the smart grid management as low communication requirements, low computational load and data privacy. These advantages are identified as being of great importance in the development of such grids [23], [24]. Another advantage of a self-organized multiagent system is the ease of introducing a local energy management when distributed energy resources are available. The rules are not strict because the agents adapt between themselves so that they can pay attention to local resources as storage or renewable energy without reporting to any central agent. Following a similar procedure, MuFCO algorithm may be used in other distributed multiagent system applications, as distributed computing, coordination of fleet of vehicles, or data networks.

\section{References}

[1] Y. Cao, W. Yu, W. Ren, and G. Chen, "An overview of recent progress in the study of distributed multi-agent coordination," IEEE Trans. Ind. Informat., vol. 9, no. 1, pp. 427-438, Feb. 2013.

[2] W. Yu, G. Chen, Z. Wang, and W. Yang, "Distributed consensus filtering in sensor networks," IEEE Trans. Syst., Man, Cybern. B, Cybern., vol. 39, no. 6, pp. 1568-1577, Dec. 2009.

[3] N. Amelina, A. Fradkov, Y. Jiang, and D. Vergados, "Approximate consensus in stochastic networks with application to load balancing," IEEE Trans. Inf. Theory, vol. 61, no. 4, pp. 1739-1752, Apr. 2015.

[4] J. A. Martín H, J. De Lope, and D. Maravall, "Adaptation, anticipation and rationality in natural and artificial systems: Computational paradigms mimicking nature," Nat. Comput., vol. 8, no. 4, pp. 757-775, Dec. 2009.

[5] S. H. Strogatz and I. Stewart, "Coupled oscillators and biological synchronization," Sci. Amer, vol. 269, no. 6, pp. 102-109, Dec. 1993.

[6] L. Glass, "Synchronization and rhythmic processes in physiology," Nature, vol. 410, no. 6825, pp. 277-284, Mar. 2001.

[7] D. C. Michaels, E. P. Matyas, and J. Jalife, "Mechanisms of sinoatrial pacemaker synchronization: A new hypothesis." Circ. Res., vol. 61, no. 5, pp. $704-714,1987$.

[8] A. F. Glova, "Phase locking of optically coupled lasers," Quant. Electron., vol. 33, no. 4, pp. 283-306, 2003.

[9] Z. Néda, E. Ravasz, Y. Brechet, T. Vicsek, and A. L. Barabási, "Selforganizing processes: The sound of many hands clapping," Nature, vol. 403 , no. 6772 , pp. $849-850$, Feb. 2000.

[10] N. Chopra and M. Spong, "On exponential synchronization of kuramoto oscillators," IEEE Trans. Autom. Control, vol. 54, no. 2, pp. 353-357, Feb. 2009.

[11] M. Castillo-Cagigal, A. Brutschy, A. Gutiérrez, M. Birattari, "Temporal task allocation in periodic environments," in Swarm Intelligence, vol. 8667, M. Dorigo et al., Eds. New York, NY, USA: Springer, 2014, pp. 182-193.

[12] O. Holland, C. Melhuish, and S. E. J. Hoddell, "Convoying: Using chorusing for the formation of travelling groups of minimal agents," Robot. Auton. Syst., vol. 28, no. 2-3, pp. 207-216, Aug. 1999.

[13] A. L. Christensen, R. O'Grady, and M. Dorigo, "From fireflies to faulttolerant swarm of robots," IEEE Trans. Evol. Comput., vol. 13, no. 4, pp. 754-766, Aug. 2009.

[14] Y. Kuramoto, Chemical Oscillations, Waves, and Turbulence. New York, NY, USA: Springer, 1984.

[15] S. H. Strogatz, "From Kuramoto to Crawford: Exploring the onset of synchronization in populations of coupled oscillators," Phys. D Nonlinear Phenom., vol. 143, nos. 1-4, pp. 1-20, Sep. 2000.

[16] P. Palensky and D. Dietrich, "Demand side management: Demand response, intelligent energy systems, and smart loads," IEEE Trans. Ind. Informat., vol. 7, no. 3, pp. 381-388, Aug. 2011.

[17] G. Strbac, "Demand side management: Benefits and challenges," Energy Policy, vol. 36, no. 12, pp. 4419-4426, 2008.

[18] R. Deng, Z. Yang, M.-Y. Chow, and J. Chen, "A survey on demand response in smart grids: Mathematical models and approaches," IEEE Trans. Ind. Informat., vol. 11, no. 3, pp. 570-582, Jun. 2015.

[19] N. Li, L. Chen, and S. Low, "Optimal demand response based on utility maximization in power networks," in Proc. IEEE Power Energy Soc. Gen. Meeting, Jul. 2011, pp. 1-8.

[20] A.-H. Mohsenian-Rad, V. Wong, J. Jatskevich, R. Schober, and A. LeonGarcia, "Autonomous demand-side management based on game-theoretic energy consumption scheduling for the future smart grid," IEEE Trans. Smart Grid, vol. 1, no. 3, pp. 320-331, Dec. 2010.

[21] A. Vaccaro, V. Loia, G. Formato, P. Wall, and V. Terzija, "A selforganizing architecture for decentralized smart microgrids synchronization, control, and monitoring," IEEE Trans. Ind. Informat., vol. 11, no. 1, pp. 289-298, Feb. 2015.

[22] M. López-Ibáńez, J. Dubois-Lacoste, T. Stützle, and M. Birattari, "The irace package, iterated race for automatic algorithm configuration," IRIDIA, Université Libre de Bruxelles, Belgium, Tech. Rep. TR/IRIDIA/2011-004, 2011 [Online]. Available: http://iridia.ulb.ac. be/irace/ 